Intern. J. Neuroscience, 115:1165-1173, 2005

Copyright $\odot 2005$ Taylor \& Francis Inc

ISSN: 0020-7454 / 1543-5245 online

DOI: $10.1080 / 00207450590914464$

\title{
THE FACE, BEAUTY, AND SYMMETRY: PERCEIVING ASYMMETRY IN BEAUTIFUL FACES
}

\section{W. ZAIDEL J. A. COHEN}

Department of Psychology University of California Los Angeles, California, USA

\begin{abstract}
The relationship between bilateral facial symmetry and beauty remains to be clarified. Here, straight head-on photographs of "beautiful" faces from the collections of professional modeling agencies were selected. First, beauty ratings were obtained for these faces. Then, the authors created symmetrical left-left and right-right composites of the beautiful faces and asked a new group of subjects to choose the most attractive pair member. "Same" responses were allowed. No difference between the left-left and right-right composites was revealed but significant differences were obtained between "same" and the leftleft or right-right. These results show that subjects detected asymmetry in beauty and suggest that very beautiful faces can be functionally asymmetrical.
\end{abstract}

Keywords attractiveness, brain laterality, evolution, hemispheric specialization, mate choice, sexual selection

\section{INTRODUCTION}

In animal biological domains perfect left-right symmetry is regarded as an indicator of fitness and high genetic quality. Asymmetry is assumed to signal low resistance to disease and susceptibility to parasites, both of which are undesirable heritable traits (Cronin, 1992; Hamilton et al., 1990). Advertising

Received 29 September 2004

Address correspondence to Dr. Dahlia W. Zaidel, Department of Psychology, UCLA, Box 951563, Los Angeles, CA 90095-1563, USA. E-mail: dahliaz@ucla.edu 
bilateral symmetry signals quality genes and offspring survival (Moller \& Miller, 1994). According to this general biological view, preference for potential mates is thus enhanced if bilateral symmetry is present. Behavioral studies in some animals suggest that animal brains are selectively neuronally attuned to perception of symmetry (Moller, 1995) and visual perceptual mechanisms are probably in place to detect deviations from symmetry (Swaddle, 1999).

The biological situation in humans is different from animals because humans possess multiple skeletal and anatomical asymmetries (Skvarilova, 1994) as well an anatomically and functionally asymmetrical brain. From early on, in typical interactions human infants gaze on adults who display subtle facial asymmetries. Functional asymmetries in human faces have been reported for emotions (Richardson et al., 2000; Wylie \& Goodale, 1988; Zaidel et al., 1995), both positive and negative (Benson \& Laskin, 2001), resemblance (Chen et al., 1997), and attractiveness (Zaidel, et al., 1995). Structural asymmetries have long been described by craniofacial and dental researchers (Vig \& Hewitt, 1975; Peck et al., 1991; Ferrario et al., 1993a, 1993b, 1995, 1994, 2003; Skvarilova, 1994), and have been depicted in the realistic art of ancient Greece and Egypt (Peck \& Peck, 1995; Peck et al., 1991). Moreover, in human fetal life, brain and craniofacial morphogenesis are closely related (Slavkin, 1995). These functional, soft-tissue, and anatomical asymmetries are subtle and are not easily discerned in ordinary interactions and viewing. Dysmorphic facial features are known to be associated with specific neurological disorders such as Down's, Fragile X, Williams', and velo-cardio-facial syndromes. Subtle abnormalities have recently been reported in schizophrenia as well (Gelowitz et al., 2002; Hennessy et al., 2004; Waddington et al., 1999). Gross facial asymmetries are unattractive, of course, but the thickness of the dividing line between the natural asymmetry and abnormal asymmetry has not yet been determined. The facial hemi-sides, as the cerebral hemispheres, are functionally asymmetric, although not surprisingly so given the morphogenetic link between brain and craniofacial appearance.

Previously published studies on functional facial asymmetries from this laboratory used faces of normal people not associated with the beauty industry and found asymmetries (Chen et al., 1997; Reis \& Zaidel, 2001; Zaidel et al., 1995). The authors now wished to determine whether or not left-right asymmetries are discernible in "beautiful" fashion models. Typically, studies investigating the bilateral symmetry issue use faces of ordinary people.

Several earlier studies have cast doubts on the relationship between beauty and symmetry (Swaddle \& Cuthill, 1995). Swaddle and Cuthill pointed out 
that manipulation of facial stimuli employing "averageness" confound assessments of the relationship. Studies that have used normal, head-on photographs and symmetrical left-left and right-right faces have also reported a weak relationship between beauty and symmetry (Knowner, 1996; Samuels et al., 1994). But morphing or digital smoothing techniques have shown strong association between symmetry and attractiveness (Grammer \& Thornhill, 1994; Rhodes et al., 1998). However, when only a hemi-face was shown subjects were able to assess attractiveness, demonstrating thereby that symmetry is not a critical determinant of beauty and that someone's beauty can be gleaned from half a face (Scheib et al., 1999). Moreover, babies viewing faces were found to be more interested in beautiful than in symmetrical faces (Rhodes et al., 2002; Samuels, et al., 1994). It would appear, then, that from birth, the human brain is more interested in facial features related to beauty than to features related to symmetry. Similarly, left-left and right-right faces that are perfectly symmetrical have been found to be less attractive than the original faces giving rise to them (Knowner, 1996). The original faces had subtle asymmetries that were perceived by the viewers. Together, the findings on this issue suggest that in humans, symmetry and attractiveness are not one and the same.

Unlike the authors' previous experiments, this one used faces photographed by others. The faces were chosen from previously existing collections in modeling agencies. Because the faces were not photographed by the authors, it could not be ruled out that lateral mirror reversals were applied in some of them. Such reversals would make it impossible to validly know whether or not there are actual hemi-face differences in the appearance of beauty. However, reversals would not interfere with conclusions regarding "same" decisions (which subjects were allowed to provide when they judged that there was no difference between left-left and right-right composites). The authors thus focused on determining if participants perceived any differences in the viewed faces, particularly in "same" responses.

\section{METHOD}

\section{Participants}

The participants were right-handed undergraduate students in introductory psychology courses at the University of California, Los Angeles (UCLA). They participated in order to receive partial course credit. Part A: For the beauty ratings of the model's faces, we had 27 participants in group one (15 
females, 12 males) and 21 new participants in group two (14 females, 7 males). Group one rated the original views of the photographs and group two rated the mirror reversed view of these photographs. Part B: For deciding which member of the pair of left-left and right-right faces was more attractive, or if there was no difference in attractiveness, there were 26 new subjects (14 females, 12 males).

\section{Stimuli}

There were a total of 36 straight, head-on photographed faces of models (20 of women and 16 of men) earning money in the beauty industry based on their looks. They were not well-known or famous and their faces were, by and large, unfamiliar to the participants. The photographs were obtained from several modeling agencies in the Los Angeles area. Thirty-five of the faces were Caucasian and one was Asian (a female). Their age range was approximately 15 to 35 years, and all of the faces had "natural" expressions. The photographs were digitized and converted to grayscale on a Macintosh computer. Part A of this study used these faces in order to obtain attractiveness rating. Part B used Macintosh software to divide each photographed face in half down the midline, in the midsagittal axis, and created a mirror image for each half. Then, the original half and its mirror image were seamlessly aligned side by side so as to have a normal looking, yet perfectly symmetrical fullface. Thus, each photographed face gave rise to a pair of faces consisting of a left-left and a right-right composite.

\section{Procedures}

Stimuli were viewed on a Macintosh computer and all responses were made through a key press directly on the computer keyboard. Participants were tested individually. Part A: Participants were asked to rate each face on a 5point attractiveness scale where " 1 " was completely unattractive and " 5 " was very attractive. A score of " 3 " indicated mid-way between very unattractive to very attractive. A "2" indicated not quite very unattractive and a "4" indicated almost very attractive. In all, participants viewed a series of 74 faces. Of those, 36 were of the models and the rest were of ordinary faces from the previously published studies from the lab of DWZ (Chen et al., 1997; Reis \& Zaidel, 2001; Zaidel et al., 1995). The faces from these two collections were intermixed within the series. Exposure duration of each face 
was $7 \mathrm{~s}$. To ensure that the lighting was symmetrical in the photographs, the faces were presented to one group in the original view (as described under Participants) and to another group in the mirror lateral view.

Part B: When pairs of left-left and right-right faces were viewed, a different group of participants was tested. They viewed a sequential presentation of 36 pairs (created from the faces of the models) for exposure duration of $10 \mathrm{~s}$ per pair. For each trial participants were asked to decide which member was more attractive or if there was no difference in attractiveness between the two members ("same" response).

\section{RESULTS}

For part A, the mean attractiveness rating for each of the faces was calculated. There was no significant difference between the original and mirror-reversed views. The participants judged the faces of the models to be much more attractive than the ordinary faces $(t(72)=11.7, p<.0001)$ used in the previous studies from this laboratory (Chen et al., 1997; Reis \& Zaidel, 2001; Zaidel, et al., 1995).

For Part B, the mean preference for left-left, right-right, and "same" was determined for each subject. These were analyzed by a repeated measures ANOVA with a within subjects factor of Face Sex (women, men) and Face Side (left-left, right-right, same). Figure 1 summarizes the results.

The ANOVA revealed a significant main effect for Face Side $(F(2,50)$ $=44.23, p<.00001$ ). An analysis of simple main effects using $t$-tests (Bonferroni adjustment was set at $p<.008$ ) showed no significant difference between left-left and right-right, regardless of Face $\operatorname{Sex}(p>.5)$, whereas "same" responses were significantly lower than either left-left, $t(25)=6.12, p<$ .0001 or right-right, $t(25)=5.29, p<.0001$, in faces of women. In male faces "same" responses were similarly significantly different, $t(25)=8.23$, $p<.0001$ for left-left and $t(25)=6.40, p<.0001$ for right-right. (With the Bonferroni adjustment for multiple comparisons, significance level was set at $p<.008$.) There was no significant interaction and no other main effect.

\section{DISCUSSION}

If beauty is closely linked to bilateral symmetry, then perfectly symmetrical left-left and right-right faces should be judged as being equally attractive. This is not what was found. Unlike the present study, previous publications 


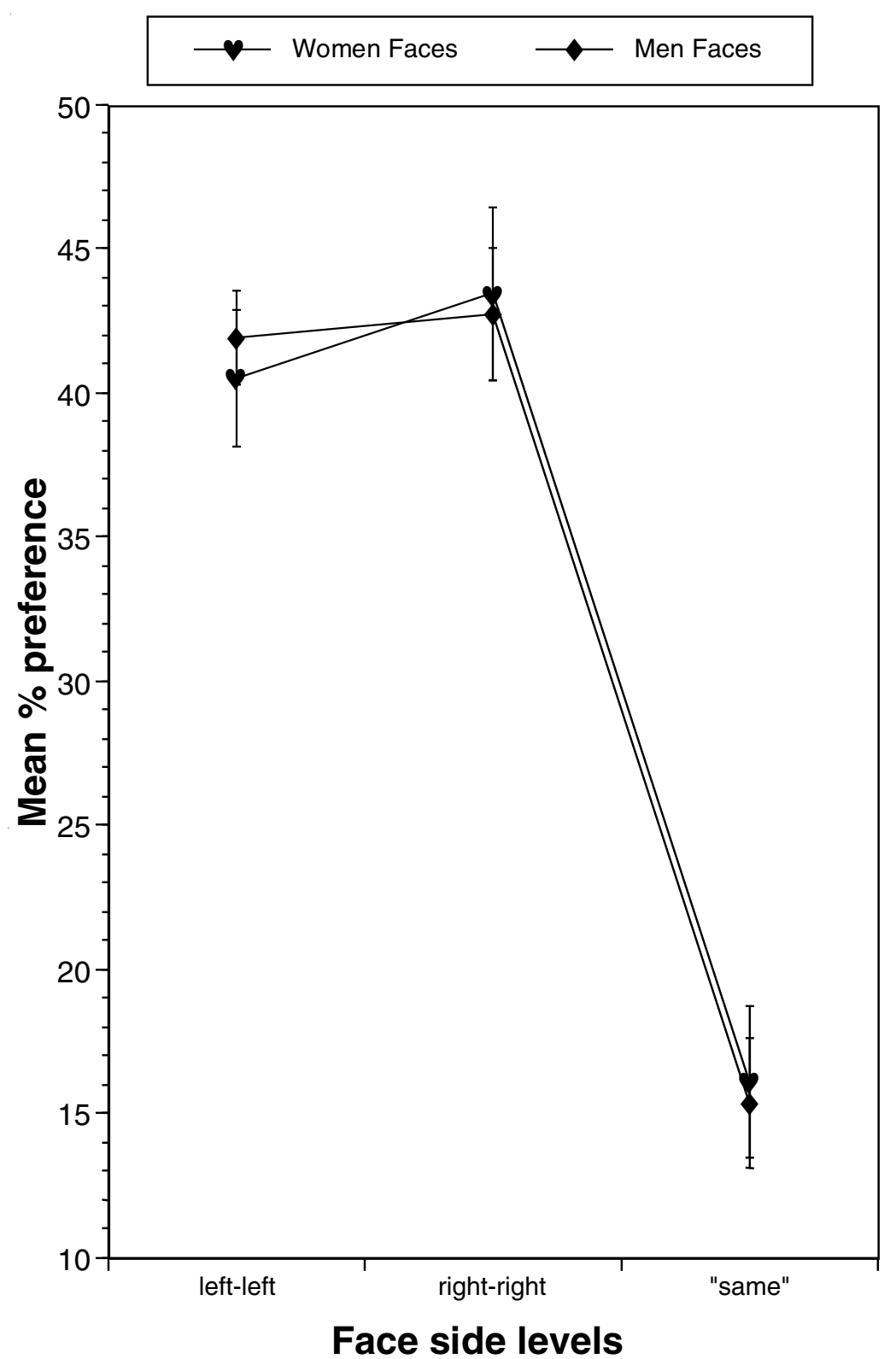

Figure 1. Summary of mean percent preference for left-left, right-right, and "same" of beautiful faces. Error bars represent standard error of the mean. The "same" responses were given when subjects judged that the left-left and right-right faces were not different from each other. This graph shows clearly there were very few "same" responses. 
from this laboratory used ordinary faces as stimuli in experiments on the functional (a)symmetry status of the face (Chen et al., 1997; Deblieck \& Zaidel, 2003; Reis \& Zaidel, 2001; Zaidel et al., 2003; Zaidel, et al., 1995). The previous findings revealed lateral facial organization for attractiveness, particularly in faces of women. Here, this issue was investigated in photographs of beautiful faces. Although they were selected from modeling agencies' photo collections, beauty was also assessed by an independent group of participants who gave the faces high attractiveness ratings. It was found that when participants compared the left-left and right-right faces they did not judge them to be the same with regard to attractiveness. This finding suggests that participants perceived subtle lateral differences in beauty even in this series of highly attractive faces.

In the previous study (Zaidel et al., 1995), subjects significantly rated the right-right composite of ordinary women's faces as being more attractive than the left-left, whereas men's right-right versus left-left was not significantly different. Here, with these beautiful faces, subjects were nearly equally split in their beauty decision for both sexes when comparing the left-left and the right-right to each other. This implies that physical markers of beauty, whatever they may be, are visible in each of the half-sides. These markers were perceived and affected the decision of the observers.

The specific phenotypic facial features of beauty remain to be identified and their spatial relationship within the facial frame should be clarified with future research. However, left-right bilateral symmetry has always been assumed to be a major contributing feature in perceptual assessment of beauty. The invisible demarcating line, or even range, separating beautiful from nonbeautiful is also unknown. Such an imaginary cut-off nevertheless does seem to play a role when participants compare the attractiveness of bilaterally symmetric laboratory constructed left-left and right-right faces. This is seen when comparing the results from the present study with the strong asymmetric appearance of attractiveness in earlier reports from this laboratory with ordinary faces (Zaidel, et al., 1995). In the present study, the phenotypic appearance of beauty was dominant in the perceptual assessment and seems to have interfered in hemi-face distinction. It did not obscure, however, the presence of some asymmetry because only a small fraction of the responses were for "same".

\section{REFERENCES}

Benson, K. J., \& Laskin, D. M. (2001). Upper lip asymmetry in adults during smiling. Journal of Oral Maxillofacial Surgery, 59, 396-398. 
Chen, A. C., German, C., \& Zaidel, D. W. (1997). Brain asymmetry and facial attractiveness: Beauty is not simply in the eye of the beholder. Neuropsychologia, 35, 471-476.

Cronin, H. (1992). The ant and the peacock. Cambridge: Cambridge University Press.

Deblieck, C., \& Zaidel, D. W. (2003). Hemifield memory for attractiveness. International Journal of Neuroscience, 113, 931-941.

Ferrario, V. F., Sforza, C., Miani, A. J., \& Tartaglia, G. (1993a). Craniofacial morphometry by photographic evaluations. American Journal of Orthodontics and Dentofacial Orthopedics, 101, 327-337.

Ferrario, V. G., Sforza, C., Pizzini, G., Vogel, G., \& Miani, A. (1993b). Sexual dimorphism in the human face assessed by euclidean distance matrix analysis. Journal of Anatomy, 183, 593-600.

Ferrario, V. F., Sforza, C., Pogio, C. E., \& Tartaglia, G. (1994). Distance from symmetry: A three-dimensional evaluation of facial asymmetry. Journal of Oral Maxillofacial Surgery, 52, 1126-1132.

Ferrario, V. F., Sforza, C., Miani, A., Jr., \& Serrao, G. (1995). A three-dimensional evaluation of human facial asymmetry. Journal of Anatomy, 186 (Pt. 1), 103110.

Ferrario, V. F., Sforza, C., Dellavia, C., Tartaglia, G. M., Colombo, A., \& Caru, A. (2003). A quantitative three-dimensional assessment of soft tissue facial asymmetry of cleft lip and palate adult patients. Journal of Craniofacial Surgery, 14, 739-46.

Gelowitz, D. L., Rakic, P., Goldman-Rakic, P. S., \& Selemon, L. D. (2002). Craniofacial dysmorphogenesis in fetally irradiated nonhuman primates: Implications for the neurodevelopmental hypothesis of schizophrenia. Biological Psychiatry, $52,716-720$.

Grammer, K., \& Thornhill, R. (1994). Human (homo sapiens) facial attractiveness and sexual selection: The role of symmetry and averageness. Journal of Comparative Psychology, 108, 233-242.

Hamilton, W. D., Axelrod, R., \& Tanese, R. (1990). Sexual reproduction as an adaptation to resist parasites (a review). Proceedings of the National Academy of Sciences, USA, 87, 3566-3573.

Hennessy, R. J., Lane, A., Kinsella, A., Larkin, C., O'Callaghan, E., \& Waddington, J. L. (2004). 3D morphometrics of craniofacial dysmorphology reveals sex-specific asymmetries in schizophrenia. Schizophrenia Research, 67(2-3), 261-268.

Knowner, R. (1996). Facial asymmetry and attractiveness judgment in developmental perspective. Journal of Experimental Psychology, 22, 662-675.

Moller, A. P. (1995). Bumblebee preference for symmetrical flowers. Proceedings of the National Academy of Sciences, USA, 92, 2288-2292.

Moller, A. P., \& Miller, A. P. (1994). Sexual selection and the barn swallow. Oxford: Oxford University Press.

Peck, S., \& Peck, L. (1995). Selected aspects of the art and science of facial esthetics. Seminars in Orthodontics, 1, 105-126. 
Peck, S., Peck, L., \& Kataia, M. (1991). Skeletal asymmetry in esthetically pleasing faces. The Angle Orthodontist, 61, 43-48.

Reis, V. A., \& Zaidel, D. W. (2001). Functional asymmetry in the human face: Perception of health in the left and right sides of the face. Laterality, 6, 225-231.

Rhodes, G., K., G., Jeffery, L., Dziurawiec, S., \& Clark, A. (2002). Are average and symmetric faces attractive to infants? Discrimination and looking preferences. Perception, 31, 315-321.

Rhodes, G., Proffitt, F., Grady, J. M., \& Sumich, A. (1998). Facial symmetry and the perception of beauty. Psychonomic Bulletin and Review, 5, 659-669.

Richardson, C. K., Bowers, D., Bauer, R. M., Heilman, K. M., \& Leonard, C. M. (2000). Digitizing the moving face during dynamic displays of emotion. Neuropsychologia, 38, 1028-1039.

Samuels, C. A., Butterworth, G., Roberts, T., Graupner, L., \& Hole, G. (1994). Facial aesthetics: Babies prefer attractiveness to symmetry. Perception, 23, 823-831.

Scheib, J. E., Gangestad, S. W., \& Thornhill, R. (1999). Facial attractiveness, symmetry and cues of good genes. Proceedings of the National Academy of Sciences, USA, 266, 1913-1917.

Skvarilova, B. (1994). Facial asymmetry: An X-ray study. Acta Chirurgiae Plasticae, 36, 89-91.

Slavkin, H. C. (1995). Molecular biology experimental strategies for craniofacial-oraldental dysmorphology. Connective Tissue Research, 32, 233-239.

Swaddle, J. P. (1999). Visual signalling by asymmetry: A review of perceptual processes. Proceedings of the Royal Society, London, B, 354, 1383-1393.

Swaddle, J. P., \& Cuthill, J. C. (1995). Asymmetry and human facial attractiveness: Symmetry may not always be beautiful. Proceedings of the Royal Society, London, B, 261, 111-116.

Vig, P. S., \& Hewitt, A. B. (1975). Asymmetry of the human facial skeleton. The Angle Orthodontist, 45, 125-129.

Waddington, J. L., Lane, A., Scully, P., Meagher, D., Quinn, J., Larkin, C., \& O’Callaghan, E. (1999). Early cerebro-craniofacial dysmorphogenesis in schizophrenia: A lifetime trajectory model from neurodevelopmental basis to "neuroprogressive" process. Journal of Psychiatric Research, 33, 477-489.

Wylie, D. R., \& Goodale, M. A. (1988). Left sided oral asymmetries in spontaneous but not posed smiles. Neuropsychologia, 26, 823-832.

Zaidel, D. W., Bava, S., \& Reis, V. A. (2003). Relationship between facial asymmetry and judging trustworthiness in faces. Laterality, 8, 225-232.

Zaidel, D. W., Chen, A. C., \& German, C. (1995). She is not a beauty even when she smiles: Possible evolutionary basis for a relationship between facial attractiveness and hemispheric specialization. Neuropsychologia, 33, 649-655. 